TRANSACTIONS OF THE

AMERICAN MATHEMATICAL SOCIETY

Volume 364, Number 8, August 2012, Pages 3993-4010

S 0002-9947(2012)05403-1

Article electronically published on March 21, 2012

\title{
LOWER BOUNDS FOR NORMS OF PRODUCTS OF POLYNOMIALS VIA BOMBIERI INEQUALITY
}

\author{
DAMIÁN PINASCO
}

\begin{abstract}
In this paper we give a different interpretation of Bombieri's norm. This new point of view allows us to work on a problem posed by Beauzamy about the behavior of the sequence $S_{n}(P)=\sup _{Q_{n}}\left[P Q_{n}\right]_{2}$, where $P$ is a fixed $m$-homogeneous polynomial and $Q_{n}$ runs over the unit ball of the Hilbert space of $n$-homogeneous polynomials. We also study the factor problem for homogeneous polynomials defined on $\mathbb{C}^{N}$ and we obtain sharp inequalities whenever the number of factors is no greater than $N$. In particular, we prove that for the product of homogeneous polynomials on infinite dimensional complex Hilbert spaces our inequality is sharp. Finally, we use these ideas to prove that any set $\left\{z_{k}\right\}_{k=1}^{n}$ of unit vectors in a complex Hilbert space for which $\sup _{\|z\|=1}\left|\left\langle z, z_{1}\right\rangle \cdots\left\langle z, z_{n}\right\rangle\right|$ is minimum must be an orthonormal system.
\end{abstract}

\section{INTRODUCTION}

Let $P_{1}, \ldots, P_{n}$ be polynomials defined on $\mathbb{K}^{N}$, where $\mathbb{K}=\mathbb{R}$ or $\mathbb{C}$, and suppose that we have a norm $\|\cdot\|$ defined on the space of polynomials. The problem of finding a constant $M$, depending only on the degrees of $P_{1}, \ldots, P_{n}$, such that

$$
\left\|P_{1}\right\| \cdots\left\|P_{n}\right\| \leq M\left\|P_{1} \cdots P_{n}\right\|
$$

and other questions concerning inequalities for the norms of factors of a given polynomial were studied by many authors: G. Aumann [3], B. Beauzamy, E. Bombieri, P. Enflo and H. L. Montgomery 9, B. Beauzamy and P. Enflo [11, P. B. Borwein [15, D. W. Boyd [17, 18, 19], A. O. Gel'fond [24, H. Kneser [26], K. Mahler [28, 29] and I. E. Pritsker and S. Ruscheweyh [35, 36] among others.

For example, for polynomials in one complex variable endowed with the supremum norm over the unit disk, D. W. Boyd [19] proved that

$$
\left\|P_{1}\right\| \cdots\left\|P_{n}\right\| \leq C_{n}^{m}\left\|P_{1} \cdots P_{n}\right\|,
$$

where the polynomial $P_{1} \cdots P_{n}$ has degree $m$ and the exact value of the constant $C_{n}$ is

$$
C_{n}=\exp \left(\frac{n}{\pi} \int_{0}^{\pi / n} \log (2 \cos (t / 2)) d t\right)
$$

Received by the editors May 5, 2010 and, in revised form, June 15, 2010.

2010 Mathematics Subject Classification. Primary 30C10, 12D05, 26D05, 46G25.

Key words and phrases. Bombieri's inequality, Bombieri's norm, Gaussian measure, plank problem, polynomial, product of linear functionals, uniform norm inequalities.

This work was partially supported by ANPCyT PICT 05 17-33042.

(C)2012 American Mathematical Society Reverts to public domain 28 years from publication 
This inequality, which is an improvement of earlier results of A. O. Gel'fond 24] and K. Mahler [28, is asymptotically sharp as $m \rightarrow \infty$.

Working with multivariate polynomials and different norms related to the coefficients of the polynomials, B. Beauzamy et al. [9] and K. Mahler [29] gave estimates for the constant $M$ in inequality (1.1). For instance, in 9], the authors defined a norm on the space of $m$-homogeneous polynomials on $\mathbb{K}^{N}$ by

$$
[P]_{2}=\left(\sum_{|\alpha|=m} \frac{\alpha !}{m !}\left|a_{\alpha}\right|^{2}\right)^{1 / 2},
$$

where $\alpha=\left(\alpha_{1}, \ldots, \alpha_{N}\right) \in \mathbb{N}^{N}$ is a multi-index, $|\alpha|=\alpha_{1}+\cdots+\alpha_{N}$ and $P$ has the monomial expansion $P\left(z_{1}, \ldots, z_{N}\right)=\sum_{|\alpha|=m} a_{\alpha} z_{1}^{\alpha_{1}} \cdots z_{N}^{\alpha_{N}}$. For this norm, which is known as Bombieri's norm, they proved the following inequality: let $P, Q$ be homogeneous polynomials of degrees $m, n$ respectively; then

$$
[P]_{2}[Q]_{2} \leq \sqrt{\frac{(m+n) !}{m ! n !}}[P Q]_{2} .
$$

Associated with inequality (1.1) we have the problem of finding

$$
I(P)=\inf \{\|P Q\|:\|Q\|=1\} \quad \text { and } \quad S(P)=\sup \{\|P Q\|:\|Q\|=1\} .
$$

This problem and other questions related to different restrictions over $P$ and $Q$ were studied by several authors. For example, B. Beauzamy [7, B. Beauzamy, J.-L. Frot and C. Millour [12] and B. Reznick 39] dealt with the problem of finding

$$
\inf \left\{[P Q]_{2}:[P]_{2}=[Q]_{2}=1\right\} \quad \text { and } \sup \left\{[P Q]_{2}:[P]_{2}=[Q]_{2}=1\right\} .
$$

There are finite dimensional variations of this problem. E. Bombieri and J. Vaaler [14, J. D. Donaldson and Q. I. Rahman [21] and J.-P. Kahane 25] studied the quantities

$$
\inf \{\|P Q\|:\|Q\|=1, \operatorname{deg}(Q)=n\} \text { and } \sup \{\|P Q\|:\|Q\|=1, \operatorname{deg}(Q)=n\}
$$

for different norms depending on the coefficients of the polynomials.

A way to compute $[P Q]_{2}$ using the eigenvalues of a matrix associated to homogeneous polynomials $P$ and $Q$ was shown by B. Beauzamy [6]. Therefore, if $\mathcal{P}\left({ }^{m} \mathbb{C}^{N}\right)$ denotes the space of $m$-homogeneous polynomials on $\mathbb{C}^{N}$, given $P \in \mathcal{P}\left({ }^{m} \mathbb{C}^{N}\right)$, the problem of finding the numbers

$$
I_{n}(P)=\inf \left\{[P Q]_{2}:[Q]_{2}=1, Q \in \mathcal{P}\left({ }^{n} \mathbb{C}^{N}\right)\right\}
$$

and

$$
S_{n}(P)=\sup \left\{[P Q]_{2}:[Q]_{2}=1, Q \in \mathcal{P}\left({ }^{n} \mathbb{C}^{N}\right)\right\}
$$

was theoretically solved. The author also showed that if $\operatorname{deg}(P)>0$, then the sequence $I_{n}(P) \rightarrow 0$. However, it seems to be difficult to characterize the behavior of $S_{n}(P)$ using those techniques. For example, if $P(z)=\langle z, a\rangle^{m}$, then $S_{n}(P)=[P]_{2}$ for all $n \in \mathbb{N}$, but for the polynomial $P\left(z_{1}, z_{2}\right)=z_{1} z_{2}, \lim _{n \rightarrow \infty} S_{n}(P)=1 / 2<$ $1 / \sqrt{2}=[P]_{2}$. The same problem was studied independently by B. Reznick [39]. In Section 3 we will prove that $\lim _{\sup _{n \rightarrow \infty}} S_{n}(P)=\|P\|_{\mathcal{P}}$.

Let us recall that the space of continuous $m$-homogeneous polynomials on a Banach space $E$, denoted by $\mathcal{P}\left({ }^{m} E\right)$, is a Banach space under the uniform norm $\|P\|_{\mathcal{P}}=\sup _{\|z\|_{E}=1}|P(z)|$. Considering this norm, inequality (1.1) was studied for 
polynomials defined on infinite dimensional Banach spaces. For instance, R. Ryan and B. Turett 38 gave bounds for the special case where the polynomials $\left\{P_{i}\right\}_{i=1}^{n}$ are continuous linear functionals on $E$. Moreover, C. Benítez, Y. Sarantopoulos and A. Tonge [13] proved that if $P_{i}$ has degree $k_{i}$ for $1 \leq i \leq n$, then inequality (1.1) holds with constant

$$
M=\frac{\left(k_{1}+\cdots+k_{n}\right)^{\left(k_{1}+\cdots+k_{n}\right)}}{k_{1}^{k_{1}} \cdots k_{n}^{k_{n}}}
$$

for any complex Banach space. The authors also showed an example on $\ell_{1}$ for which the equality prevails. However, for many spaces it is possible to improve this bound. In [16], C. Boyd and R. Ryan proved that, on complex Hilbert spaces,

$$
M=\frac{\left(k_{1}+\cdots+k_{n}\right) !}{k_{1} ! \cdots k_{n} !}
$$

is more accurate.

We will be concerned with the study of inequality (1.1) for homogeneous polynomials defined on a (finite or infinite dimensional) complex Hilbert space $H$. In Section 4 we will prove that

$$
\left\|P_{1}\right\|_{\mathcal{P}} \cdots\left\|P_{n}\right\|_{\mathcal{P}} \leq \sqrt{\frac{\left(k_{1}+\cdots+k_{n}\right)^{\left(k_{1}+\cdots+k_{n}\right)}}{k_{1}^{k_{1}} \cdots k_{n}^{k_{n}}}}\left\|P_{1} \cdots P_{n}\right\|_{\mathcal{P}},
$$

where $P_{i} \in \mathcal{P}\left({ }^{k_{i}} H\right)$ for $1 \leq i \leq n$. We also prove that this is a sharp inequality whenever the number of factors is no greater than $\operatorname{dim}(H)$. We derive sharp inequalities for products of continuous symmetric multilinear forms on complex Hilbert spaces and using complexification techniques (see [32]) we present inequalities for real Hilbert spaces.

Inequality (1.1) has been widely studied when $P_{1}, \ldots, P_{n}$ are bounded linear functionals on Hilbert spaces. In [13, the authors made the following conjecture: given $n$ unit vectors $\left\{x_{k}\right\}_{k=1}^{n}$ in $\mathbb{R}^{n}$, then

$$
\sup _{\|x\|_{\mathbb{R}^{n}=1}}\left|\left\langle x, x_{1}\right\rangle \cdots\left\langle x, x_{n}\right\rangle\right| \geq n^{-n / 2},
$$

and equality holds if and only if $\left\{x_{k}\right\}_{k=1}^{n}$ is an orthonormal system. This conjecture was also discussed by A. E. Litvak, V. D. Milman, and G. Schechtman [27. A positive answer for $n=1,2,3,4$ and 5 was given by A. Pappas and S. G. Révész 33. However, the remaining cases are still open problems. See for example V. A. Anagnostopoulos and S. G. Révész [1, P. E. Frenkel [22], J. C. García-Vázquez and R. Villa [23], M. Matolcsi [30, 31] and S. G. Révész and Y. Sarantopoulos [37] for different approaches and related problems.

In the complex setting, K. Ball [4] proved a stronger result than inequality (1.3), which is known as "the complex plank problem for Hilbert spaces". A few years before K. Ball's result, J. Arias-de-Reyna [2] proved an inequality which is the complex analogue of (1.3). In his paper, the author showed that $n^{-n / 2}$ is the best possible constant, because for any orthonormal system $\left\{z_{k}\right\}_{k=1}^{n}$ it follows that

$$
\sup _{\|z\|_{\mathbb{C}^{n}=1}}\left|\left\langle z, z_{1}\right\rangle \cdots\left\langle z, z_{n}\right\rangle\right|=n^{-n / 2} .
$$


Unfortunately, his proof did not allow him to show if this orthogonal condition on the set $\left\{z_{k}\right\}_{k=1}^{n}$ is necessary to achieve the minimum value. In the last section, using inequality (1.2), we will be able to prove that a set $\left\{z_{k}\right\}_{k=1}^{n}$ of unit vectors in a complex Hilbert space $H$ for which $\sup _{\|z\|_{H}=1}\left|\left\langle z, z_{1}\right\rangle \cdots\left\langle z, z_{n}\right\rangle\right|$ is minimum must be an orthonormal system.

\section{Polynomials And Bombieri's nORM}

Let us fix some standard notation. From now on, $\alpha$ will be a multi-index, and we will denote $|\alpha|=\alpha_{1}+\cdots+\alpha_{N}$ and $\alpha !=\alpha_{1} ! \cdots \alpha_{N}$ !. For $z \in \mathbb{C}^{N}$, $z^{\alpha}$ will stand for $z_{1}^{\alpha_{1}} \cdots z_{N}^{\alpha_{N}}$. As usual, the space of $m$-homogeneous polynomials defined on $\mathbb{C}^{N}$ will be denoted by $\mathcal{P}\left({ }^{m} \mathbb{C}^{N}\right)$. Given $P \in \mathcal{P}\left({ }^{m} \mathbb{C}^{N}\right)$, we will write $P(z)=\sum_{|\alpha|=m} a_{\alpha} z^{\alpha}$.

We will be concerned with the study of two different norms for $P$ : the uniform norm $\|P\|_{\mathcal{P}}=\sup _{\|z\|=1}|P(z)|$ and Bombieri's norm, defined in [9] as

$$
[P]_{2}=\left(\sum_{|\alpha|=m} \frac{\alpha !}{m !}\left|a_{\alpha}\right|^{2}\right)^{1 / 2} .
$$

Canonically associated with Bombieri's norm there exists an inner product (see [10], 39]). If $P, Q \in \mathcal{P}\left({ }^{m} \mathbb{C}^{N}\right), P(z)=\sum_{|\alpha|=m} a_{\alpha} z^{\alpha}$ and $Q(z)=\sum_{|\alpha|=m} b_{\alpha} z^{\alpha}$, then this inner product is

$$
[P, Q]_{(m)}=\sum_{|\alpha|=m} \frac{\alpha !}{m !} a_{\alpha} \overline{b_{\alpha}} .
$$

The following theorem, proved by Beauzamy et al. [9, is crucial for our purposes.

Theorem 2.1 (Bombieri's inequality [9, Theorem 1.2]). Let $P, Q$ be homogeneous polynomials of degrees $m, n$ respectively. Then

$$
[P Q]_{2} \geq \sqrt{\frac{m ! n !}{(m+n) !}}[P]_{2}[Q]_{2} .
$$

Corollary 2.2. Let $P_{i} \in \mathcal{P}\left({ }^{k_{i}} \mathbb{C}^{N}\right)$ for $1 \leq i \leq n$. Then

$$
\left[P_{1} \cdots P_{n}\right]_{2} \geq \sqrt{\frac{k_{1} ! \cdots k_{n} !}{\left(k_{1}+\ldots+k_{n}\right) !}}\left[P_{1}\right]_{2} \cdots\left[P_{n}\right]_{2} .
$$

Proof. The proof is immediate by induction on $n$, since

$$
\begin{aligned}
{\left[P_{1} \cdots P_{n} P_{n+1}\right]_{2} } & \geq \sqrt{\frac{\left(k_{1}+\cdots+k_{n}\right) ! k_{n+1} !}{\left(k_{1}+\ldots+k_{n+1}\right) !}}\left[P_{1} \cdots P_{n}\right]_{2}\left[P_{n+1}\right]_{2} \\
& \geq \sqrt{\frac{k_{1} ! \cdots k_{n} ! k_{n+1} !}{\left(k_{1}+\ldots+k_{n+1}\right) !}}\left[P_{1}\right]_{2} \cdots\left[P_{n}\right]_{2}\left[P_{n+1}\right]_{2} .
\end{aligned}
$$

Bombieri's inequality was also proved using differential identities based on the following property (see [10, Lemma 9] or [39]): let $P$ and $Q$ be homogeneous poly- 
nomials of degrees $m-1$ and $m$ respectively. Then

$$
\left[z_{1} P, Q\right]_{(m)}=\frac{1}{m}\left[P, \frac{\partial Q}{\partial z_{1}}\right]_{(m-1)} .
$$

B. Reznick 39 gave an alternative interpretation of $[P]_{2}$. He worked with forms of degree $m$ in $N$ variables and differential operators associated with them. Namely,

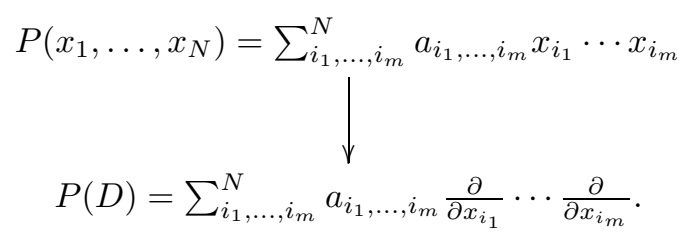

The author defined $\|P\|_{(R)}=(\operatorname{deg}(P) !)^{1 / 2}[P]_{2}$ and proved that $\|P\|_{(R)}=P(D) \bar{P}$. He also proved that $\|P Q\|_{(R)} \geq\|P\|_{(R)}\|Q\|_{(R)}$ (which is equivalent to Bombieri's inequality), where equality holds if and only if $P$ and $Q$ are unitarily disjoint. The problem of finding pairs of polynomials $(P, Q)$ for which $[P Q]_{2}$ is maximum or minimum was studied in [7], [8], 12] and [39].

2.1. A different point of view for Bombieri's norm. This section is devoted to presenting alternative definitions of $[P]_{2}$ and $\|P\|_{(R)}$. From this, we will obtain a different proof of the differential identity (2.1) using a simple lemma from [34.

Given a measure space $(X, \mu)$, we will write $L^{p}(\mu)$ to denote the space of measurable functions $f: X \rightarrow \mathbb{C}$ such that $\int_{X}|f|^{p} d \mu<\infty$. From now on, $\Gamma_{N}$ denotes the Gaussian measure defined on the Borel subsets of $\mathbb{C}^{N}$ by

$$
\Gamma_{N}(\Delta)=\int_{\Delta} e^{-\|z\|^{2}} \frac{d z}{\pi^{N}}
$$

where $d z$ stands for the Lebesgue measure on $\mathbb{C}^{N}$.

Lemma 2.3 ([34, Lemma 2.1]). Let $\Gamma_{N}$ be the Gaussian measure defined above. Then

$$
\int_{\mathbb{C}^{N}} z^{\alpha} \bar{z}^{\beta} d \Gamma_{N}(z)=\delta_{\alpha \beta} \alpha ! .
$$

In the sequel, $L_{m}^{2}\left(\Gamma_{N}\right)$ stands for the closure of $\operatorname{span}\left\{z^{\alpha}\right\}_{|\alpha|=m}$ in $L^{2}\left(\Gamma_{N}\right)$. The previous lemma shows that if $m \neq m^{\prime}$, then $L_{m}^{2}\left(\Gamma_{N}\right) \perp L_{m^{\prime}}^{2}\left(\Gamma_{N}\right)$.

Proposition 2.4. Given $m \in \mathbb{N}$, let $\imath:\left(\mathcal{P}\left({ }^{m} \mathbb{C}^{N}\right),[\cdot]_{2}\right) \rightarrow L_{m}^{2}\left(\Gamma_{N}\right)$ be defined by $\imath(P)=P$. Then $\|\imath(P)\|_{L^{2}\left(\Gamma_{N}\right)}=\sqrt{m !}[P]_{2}$.

Proof. Note that $|P(z)| \leq \max _{\|\omega\|=1}|P(\omega)|\|z\|^{m}=\|P\|_{\mathcal{P}}\|z\|^{m}$. Thus we have

$$
\int_{\mathbb{C}^{N}}|P(z)|^{2} e^{-\|z\|^{2}} \frac{d z}{\pi^{N}}<\infty .
$$

Consequently, the map is well defined. Since $\left\{z^{\alpha} / \sqrt{\alpha !}\right\}_{|\alpha|=m}$ is an orthonormal basis for the Hilbert space $L_{m}^{2}\left(\Gamma_{N}\right)$ and $\left\{\sqrt{m !} z^{\alpha} / \sqrt{\alpha !}\right\}_{|\alpha|=m}$ is an orthonormal basis 
for the Hilbert space $\left(\mathcal{P}\left({ }^{m} \mathbb{C}^{N}\right),[\cdot]_{2}\right)$, we conclude that $\|\imath(P)\|_{L^{2}\left(\Gamma_{N}\right)}=\sqrt{m !}[P]_{2}$ for all $P \in \mathcal{P}\left({ }^{m} \mathbb{C}^{N}\right)$.

Remark 2.5. Recall from [39] that $\|P\|_{(R)}=(\operatorname{deg}(P) !)^{1 / 2}[P]_{2}$. Thus, given any polynomial $P \in \mathcal{P}\left({ }^{m} \mathbb{C}^{N}\right)$, we have $\|P\|_{(R)}=\|P\|_{L^{2}\left(\Gamma_{N}\right)}$. We can restate Bombieri's inequality in terms of the $L^{2}\left(\Gamma_{N}\right)$ norm: given $P_{i} \in \mathcal{P}\left({ }^{k_{i}} \mathbb{C}^{N}\right)$ for $1 \leq i \leq n$, then it follows that

$$
\left\|P_{1} \cdots P_{n}\right\|_{L^{2}\left(\Gamma_{N}\right)} \geq\left\|P_{1}\right\|_{L^{2}\left(\Gamma_{N}\right)} \cdots\left\|P_{n}\right\|_{L^{2}\left(\Gamma_{N}\right)} .
$$

Now, we are able to give a different proof of (2.1). The nature of this new point of view is more analytical than the previous ones.

Lemma 2.2 in 34 gives an integral representation formula for entire functions which are in $L^{p}\left(\Gamma_{N}\right)$ for some $p>1$. We only need the following particular case:

Lemma 2.6. Let $P \in \mathcal{P}\left({ }^{m} \mathbb{C}^{N}\right)$. Then for every $z \in \mathbb{C}^{N}$,

$$
P(z)=\int_{\mathbb{C}^{N}} e^{\langle z, \omega\rangle} P(\omega) d \Gamma_{N}(\omega)
$$

We can use this integral formula to compute the directional derivatives of an $m$-homogeneous polynomial.

Proposition 2.7. Let $P \in \mathcal{P}\left({ }^{m} \mathbb{C}^{N}\right)$ and $v \in \mathbb{C}^{N}$. Then we have

$$
\frac{\partial P}{\partial v}(z)=\int_{\mathbb{C}^{N}} e^{\langle z, \omega\rangle}\langle v, \omega\rangle P(\omega) d \Gamma_{N}(\omega)
$$

Proof. Using Lemma 2.6 and the dominated convergence theorem,

$$
\begin{aligned}
\frac{\partial P}{\partial v}(z) & =\lim _{h \rightarrow 0} \int_{\mathbb{C}^{N}} \frac{e^{h\langle v, \omega\rangle}-1}{h} e^{\langle z, \omega\rangle} P(\omega) d \Gamma_{N}(\omega) \\
& =\int_{\mathbb{C}^{N}} e^{\langle z, \omega\rangle}\langle v, \omega\rangle P(\omega) d \Gamma_{N}(\omega) .
\end{aligned}
$$

Note that, in general, $\langle v, \omega\rangle P(\omega) \notin L_{m-1}^{2}\left(\Gamma_{N}\right)$ because it is not a holomorphic function. Since $\left\{L_{k}^{2}\left(\Gamma_{N}\right)\right\}_{k \in \mathbb{N}}$ are closed subspaces of $L^{2}\left(\Gamma_{N}\right)$, we have a sequence of orthogonal projections $\pi_{k}: L^{2}\left(\Gamma_{N}\right) \rightarrow L_{k}^{2}\left(\Gamma_{N}\right)$. By Proposition 2.4 we know that $\imath:\left(\mathcal{P}\left({ }^{m} \mathbb{C}^{N}\right),[\cdot]_{2}\right) \rightarrow L_{m}^{2}\left(\Gamma_{N}\right)$ is an isomorphism for all $m \in \mathbb{N}$, and since

$$
\frac{\partial P}{\partial v}(z)=\int_{\mathbb{C}^{N}} e^{\langle z, \omega\rangle} \frac{\partial P}{\partial v}(\omega) d \Gamma_{N}(\omega)
$$

we can deduce that $\pi_{m-1}(\langle v, \cdot\rangle P)=\frac{\partial P}{\partial v}$. 
Proposition 2.8 ([10, Lemma 9]). If $P \in \mathcal{P}\left({ }^{m} \mathbb{C}^{N}\right)$ and $Q \in \mathcal{P}\left({ }^{m-1} \mathbb{C}^{N}\right)$, then

$$
[\langle\cdot, v\rangle Q, P]_{(m)}=\frac{1}{m}\left[Q, \frac{\partial P}{\partial v}\right]_{(m-1)} .
$$

Proof.

$$
\begin{aligned}
\frac{1}{m}\left[Q, \frac{\partial P}{\partial v}\right]_{(m-1)} & =\frac{1}{m} \frac{1}{(m-1) !}\left\langle Q, \frac{\partial P}{\partial v}\right\rangle_{L_{m-1}^{2}\left(\Gamma_{N}\right)} \\
& =\frac{1}{m !}\left\langle Q, \pi_{m-1}(\langle v, \cdot\rangle P)\right\rangle_{L_{m-1}^{2}\left(\Gamma_{N}\right)} \\
& =\frac{1}{m !}\langle Q,\langle v, \cdot\rangle P\rangle_{L^{2}\left(\Gamma_{N}\right)} \\
& =\frac{1}{m !} \int_{\mathbb{C}^{N}} Q(\omega) \overline{\langle v, \omega\rangle P(\omega)} d \Gamma_{N}(\omega) \\
& =\frac{1}{m !} \int_{\mathbb{C}^{N}}\langle\omega, v\rangle Q(\omega) \overline{P(\omega)} d \Gamma_{N}(\omega) \\
& =[\langle\cdot, v\rangle Q, P]_{(m)} .
\end{aligned}
$$

In the following sections we present some applications of this interpretation of Bombieri's norm.

\section{A Limit PRoblem For $S_{n}(P)$}

In this section we use the relation between Bombieri's norm and $\|\cdot\|_{L^{2}\left(\Gamma_{N}\right)}$, proved in Proposition 2.4 to work on a problem originally posed by B. Beauzamy in 6 .

Letting $P \in \mathcal{P}\left({ }^{m} \mathbb{C}^{N}\right)$, we will study the behavior of the sequence of real numbers $S_{n}(P)$, defined by

$$
S_{n}(P)=\sup \left\{[P Q]_{2}:[Q]_{2}=1, Q \in \mathcal{P}\left({ }^{n} \mathbb{C}^{N}\right)\right\} .
$$

Our point of view of Bombieri's norm allows us to compute $\|\cdot\|_{L^{2}\left(\Gamma_{N}\right)}$ instead of $[\cdot]_{2}$. The main advantage of this is that we will be able to use classical tools from integration theory.

In the sequel, $\sigma_{N}$ denotes the normalized Lebesgue measure on $\partial B_{1}\left(\mathbb{C}^{N}\right)$, the unit sphere of $\mathbb{C}^{N}$. To shorten notation, we let $\partial B_{1}$ and $L_{\partial B_{1}}^{p}$ stand for $\partial B_{1}\left(\mathbb{C}^{N}\right)$ and $L^{p}\left(\partial B_{1}\left(\mathbb{C}^{N}\right), \sigma_{N}\right)$ respectively.

Remark 3.1. Since for homogeneous polynomials we have a link between $[\cdot]_{2}$ and $\|\cdot\|_{L^{2}\left(\Gamma_{N}\right)}$, we want to find a way to relate Bombieri's norm of a polynomial (or its 
powers) and the different values of its $L_{\partial B_{1}}^{r}$ norms. With this we aim to use that

$$
\lim _{r \rightarrow \infty}\|P\|_{L_{\partial B_{1}}^{r}}=\|P\|_{L_{\partial B_{1}}^{\infty}}=\sup _{z \in \partial B_{1}}|P(z)|=\|P\|_{\mathcal{P}} .
$$

In the rest of this section we need to compute the following integral for different values of $k \in \mathbb{N}$ :

$$
\int_{0}^{\infty} t^{2 k} e^{-t^{2}} 2 t d t=\int_{0}^{\infty} t^{k} e^{-t} d t=k !
$$

Lemma 3.2. Suppose that $P \in \mathcal{P}\left({ }^{m} \mathbb{C}^{N}\right)$ and $r \in \mathbb{N}$. Then

$$
\left[P^{r}\right]_{2}^{2}=\left(\begin{array}{c}
N-1+m r \\
N-1
\end{array}\right)\|P\|_{L_{\partial B_{1}}^{2 r}}^{2 r}
$$

Proof. According to Proposition 2.4 since $P^{r} \in \mathcal{P}\left({ }^{r m} \mathbb{C}^{N}\right)$ we have

$$
\left[P^{r}\right]_{2}^{2}=\frac{1}{(r m) !} \int_{\mathbb{C}^{N}}\left|P^{r}(w)\right|^{2} d \Gamma_{N}(w)
$$

Introducing polar coordinates, we find

$$
\begin{aligned}
{\left[P^{r}\right]_{2}^{2} } & =\frac{\left|\partial B_{1}\right|}{(r m) !} \int_{0}^{\infty} \int_{\partial B_{1}} \rho^{2 N-1} \rho^{2 m r} e^{-\rho^{2}}|P(\theta)|^{2 r} \frac{d \rho d \sigma_{N}(\theta)}{\pi^{N}} \\
& =\frac{2}{(N-1) !(r m) !} \int_{0}^{\infty} \int_{\partial B_{1}} \rho^{2 N-1} \rho^{2 m r} e^{-\rho^{2}}|P(\theta)|^{2 r} d \rho d \sigma_{N}(\theta) \\
& =\left(\frac{1}{(N-1) !(r m) !} \int_{0}^{\infty} \rho^{2(N-1+m r)} e^{-\rho^{2}} 2 \rho d \rho\right)\left(\int_{\partial B_{1}}|P(\theta)|^{2 r} d \sigma_{N}(\theta)\right) \\
& =\frac{(N-1+m r) !}{(N-1) !(r m) !}\|P\|_{L_{\partial B_{1}}^{2 r}}^{2 r}=\left(\begin{array}{c}
N-1+m r \\
N-1
\end{array}\right)\|P\|_{L_{\partial B_{1}}^{2 r}}^{2 r},
\end{aligned}
$$

which is the desired conclusion.

Our main result in this section is the following.

Theorem 3.3. Let $P \in \mathcal{P}\left({ }^{m} \mathbb{C}^{N}\right)$. Then $\limsup _{n \rightarrow \infty} S_{n}(P)=\|P\|_{\mathcal{P}}$. 
Proof. By Proposition 2.4, given $Q \in \mathcal{P}\left({ }^{n} \mathbb{C}^{N}\right)$ we have

$$
\begin{aligned}
{\left[P \frac{Q}{[Q]_{2}}\right]_{2}^{2} } & =\frac{[P Q]_{2}^{2}}{[Q]_{2}^{2}}=\frac{n !}{(m+n) !} \frac{\int_{\mathbb{C}^{N}}|P(w) Q(w)|^{2} d \Gamma_{N}(w)}{\int_{\mathbb{C}^{N}}|Q(w)|^{2} d \Gamma_{N}(w)} \\
& =\frac{n !}{(n+m) !} \frac{\int_{0}^{\infty} \int_{\partial B_{1}} \rho^{2 N-1} \rho^{2(n+m)} e^{-\rho^{2}}|P(\theta) Q(\theta)|^{2} d \rho d \sigma_{N}(\theta)}{\int_{0}^{\infty} \int_{\partial B_{1}} \rho^{2 N-1} \rho^{2 n} e^{-\rho^{2}}|Q(\theta)|^{2} d \rho d \sigma_{N}(\theta)} \\
& =\frac{n !(N-1+m+n) !}{(m+n) !(N-1+n) !} \frac{\int_{\partial B_{1}}|P(\theta)|^{2}|Q(\theta)|^{2} d \sigma_{N}(\theta)}{\int_{\partial B_{1}}|Q(\theta)|^{2} d \sigma_{N}(\theta)} \\
& \leq \frac{n !(N-1+m+n) !}{(m+n) !(N-1+n) !} \frac{\sup _{\zeta \in \partial B_{1}}|P(\zeta)|^{2} \int_{\partial B_{1}}|Q(\theta)|^{2} d \sigma_{N}(\theta)}{\int_{\partial B_{1}}|Q(\theta)|^{2} d \sigma_{N}(\theta)} \\
& =\frac{n !(N-1+m+n) !}{(m+n) !(N-1+n) !}\|P\|_{\mathcal{P}}^{2},
\end{aligned}
$$

which is equivalent to

$$
\left[P \frac{Q}{[Q]_{2}}\right]_{2} \leq \sqrt{\frac{n !(N-1+m+n) !}{(m+n) !(N-1+n) !}}\|P\|_{\mathcal{P}}
$$

Note that

$$
\frac{n !(N-1+m+n) !}{(m+n) !(N-1+n) !}=\frac{\prod_{j=1}^{m}(N-1+n+j)}{\prod_{j=1}^{m}(n+j)}=\frac{n^{m}+o\left(n^{m}\right)}{n^{m}+o\left(n^{m}\right)},
$$

where $o\left(n^{m}\right)$, as usual, means that $\frac{o\left(n^{m}\right)}{n^{m}} \underset{n \rightarrow \infty}{\longrightarrow} 0$. From this, we conclude that

$$
\frac{n !(N-1+m+n) !}{(m+n) !(N-1+n) !} \underset{n \rightarrow \infty}{\longrightarrow} 1 \text {. }
$$

Thus for any $\varepsilon>0$, there exists $n_{\varepsilon} \in \mathbb{N}$ such that for any $n \in \mathbb{N}, n>n_{\varepsilon}$, and any $Q \in \mathcal{P}\left({ }^{n} \mathbb{C}^{N}\right)$, we have

$$
\left[P \frac{Q}{[Q]_{2}}\right]_{2} \leq(1+\varepsilon)\|P\|_{\mathcal{P}}
$$

Hence $\limsup _{n \rightarrow \infty} S_{n}(P) \leq\|P\|_{\mathcal{P}}$. 
In order to prove the converse inequality consider the sequence of polynomials $\left\{P^{r}\right\}_{r \in \mathbb{N}}$. According to Lemma 3.2, we have

$$
\left[P \frac{P^{r}}{\left[P^{r}\right]_{2}}\right]_{2}^{2}=\frac{\left[P^{r+1}\right]_{2}^{2}}{\left[P^{r}\right]_{2}^{2}}=\frac{\left(\begin{array}{c}
N-1+m(r+1) \\
N-1
\end{array}\right)\|P\|_{L_{\partial B_{1}}^{2(r+1)}}^{2(r+1)}}{\left(\begin{array}{c}
N-1+m r \\
N-1
\end{array}\right)\|P\|_{L_{\partial B_{1}}^{2 r}}^{2 r}} .
$$

First, note that

$$
\frac{\left(\begin{array}{c}
N-1+m(r+1) \\
N-1
\end{array}\right)}{\left(\begin{array}{c}
N-1+m r \\
N-1
\end{array}\right)}=\frac{\prod_{j=1}^{m}(N-1+m r+j)}{\prod_{j=1}^{m}(m r+j)}=\frac{(m r)^{m}+o\left(r^{m}\right)}{(m r)^{m}+o\left(r^{m}\right)} \underset{r \rightarrow \infty}{\longrightarrow} 1 .
$$

Also, since for any probability space $(X, \mu)$ and any measurable function $f: X \rightarrow \mathbb{C}$ it follows that $\|f\|_{L^{p}(\mu)} \leq\|f\|_{L^{q}(\mu)}$ whenever $p \leq q$,

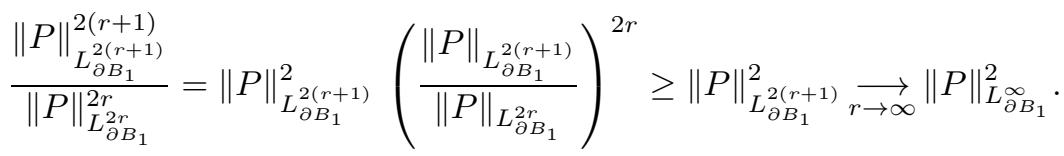

Therefore,

$$
\limsup _{r \rightarrow \infty}\left[P \frac{P^{r}}{\left[P^{r}\right]_{2}}\right]_{2}^{2} \geq\|P\|_{L_{\partial B_{1}}^{\infty}}^{2}=\|P\|_{\mathcal{P}}^{2}
$$

and we conclude that $\limsup _{n \rightarrow \infty} S_{n} \geq\|P\|_{\mathcal{P}}$.

\section{LOWER BOUNDS FOR PRODUCTS OF POLYNOMIALS ON HILBERT SPACES}

We begin this section by recalling that a continuous $m$-homogeneous polynomial from a Banach space $E$ to the scalar field $\mathbb{K}$ is a mapping $P: E \rightarrow \mathbb{K}$ for which there exists a (unique) continuous symmetric $m$-linear form $\check{P}: E^{m} \rightarrow \mathbb{K}$ such that $P(z)=\check{P}(z, \ldots, z)$ for all $z \in E$. The space of continuous symmetric $m$ linear forms $L: E^{m} \rightarrow \mathbb{K}$, denoted by $\mathcal{L}^{s}\left({ }^{m} E\right)$, is a Banach space under the norm $\|L\|_{\mathcal{L}^{s}}=\sup _{\left\|z_{j}\right\|_{E}=1}\left|L\left(z_{1}, \ldots, z_{m}\right)\right|$. Moreover, the mapping $P \mapsto \check{P}$ is an isomorphism between the Banach spaces $\left(\mathcal{P}\left({ }^{m} E\right),\|\cdot\|_{\mathcal{P}}\right)$ and $\left(\mathcal{L}^{s}\left({ }^{m} E\right),\|\cdot\|_{\mathcal{L}^{s}}\right)$. For a thorough treatment we refer the reader to [20].

Now we will turn our attention to studying inequality (1.1) for polynomials defined on Hilbert spaces. Here and subsequently, $H$ stands for an infinite dimensional complex Hilbert space and $\left\{e_{i}\right\}_{i=1}^{\infty}$ denotes a fixed orthonormal basis for $H$.

Given $P_{i} \in \mathcal{P}\left({ }^{k_{i}} H\right)$ for $1 \leq i \leq n$, we are interested in finding the optimum constant $M$, depending only on $k_{1}, \ldots, k_{n}$, for which the inequality

$$
\left\|P_{1}\right\|_{\mathcal{P}} \cdots\left\|P_{n}\right\|_{\mathcal{P}} \leq M\left\|P_{1} \cdots P_{n}\right\|_{\mathcal{P}}
$$

holds.

The following theorem presents an inequality for norms of products of polynomials on $\mathbb{C}^{N}$. The value of the proposed constant is sharp for $n \leq N$.

Theorem 4.1. Let $P_{i} \in \mathcal{P}\left(k_{i} \mathbb{C}^{N}\right)$ for $i=1, \ldots, n$. Then

$$
\left\|P_{1}\right\|_{\mathcal{P}} \cdots\left\|P_{n}\right\|_{\mathcal{P}} \leq \sqrt{\frac{\left(k_{1}+\cdots+k_{n}\right)^{\left(k_{1}+\cdots+k_{n}\right)}}{k_{1}^{k_{1}} \cdots k_{n}^{k_{n}}}}\left\|P_{1} \cdots P_{n}\right\|_{\mathcal{P}} .
$$


Proof. According to Lemma 3.2 we have

$$
\left[\left(P_{1} \cdots P_{n}\right)^{r}\right]_{2}^{2}=\left(\begin{array}{c}
N-1+r \sum_{i=1}^{n} k_{i} \\
N-1
\end{array}\right)\left\|P_{1} \cdots P_{n}\right\|_{L_{\partial B_{1}}^{2 r}}^{2 r} .
$$

Also, for $1 \leq i \leq n$,

$$
\left[P_{i}^{r}\right]_{2}^{2}=\left(\begin{array}{c}
N-1+r k_{i} \\
N-1
\end{array}\right)\left\|P_{i}\right\|_{L_{\partial B_{1}}^{2 r}}^{2 r} .
$$

Applying Bombieri's inequality, we obtain

$$
\left[P_{1}^{r}\right]_{2} \cdots\left[P_{n}^{r}\right]_{2} \leq \sqrt{\frac{\left(r \sum_{i=1}^{n} k_{i}\right) !}{\left(r k_{1}\right) ! \cdots\left(r k_{n}\right) !}}\left[\left(P_{1} \cdots P_{n}\right)^{r}\right]_{2},
$$

or equivalently

$$
\begin{aligned}
\prod_{i=1}^{n}\left\|P_{i}\right\|_{L_{\partial B_{1}}^{2 r}} & =\sqrt[2 r]{\frac{\prod_{i=1}^{n}\left[P_{i}^{r}\right]_{2}^{2}}{\prod_{i=1}^{n}\left(\begin{array}{c}
N-1+r k_{i} \\
N-1
\end{array}\right)}} \\
& \leq \sqrt[2 r]{\frac{\left(r \sum_{i=1}^{n} k_{i}\right) !\left[\left(P_{1} \cdots P_{n}\right)^{r}\right]_{2}^{2}}{\prod_{i=1}^{n}\left(r k_{i}\right) !\left(\begin{array}{c}
N-1+r k_{i} \\
N-1
\end{array}\right)}} \\
& =\sqrt[2 r]{\frac{\left(r \sum_{i=1}^{n} k_{i}\right) !\left(\begin{array}{c}
N-1+r \sum_{i=1}^{n} k_{i} \\
N-1
\end{array}\right)}{\prod_{i=1}^{n}\left(r k_{i}\right) !\left(\begin{array}{c}
N-1+r k_{i} \\
N-1
\end{array}\right)}}\left\|P_{1} \cdots P_{n}\right\|_{L_{\partial B_{1}}^{2 r}} \\
& =\sqrt[2 r]{\frac{((N-1) !)^{n-1}\left(N-1+r \sum_{i=1}^{n} k_{i}\right) !}{\prod_{i=1}^{n}\left(N-1+r k_{i}\right) !}}\left\|P_{1} \cdots P_{n}\right\|_{L_{\partial B_{1}}^{2 r}} .
\end{aligned}
$$

It is well known that given a sequence of positive real numbers $\left\{a_{r}\right\}_{r \in \mathbb{N}}$, if $\lim _{r \rightarrow \infty} \frac{a_{r+1}}{a_{r}}=L$, then $\lim _{r \rightarrow \infty} \sqrt[r]{a_{r}}=L$. For $s=\sum_{i=1}^{n} k_{i}$, let us choose

$$
a_{r}=\sqrt{\frac{((N-1) !)^{n-1}(N-1+r s) !}{\prod_{i=1}^{n}\left(N-1+r k_{i}\right) !}} .
$$

For this sequence we may compute

$$
\begin{aligned}
\frac{a_{r+1}}{a_{r}} & =\sqrt{\frac{(N-1+(r+1) s) ! \prod_{i=1}^{n}\left(N-1+r k_{i}\right) !}{(N-1+r s) ! \prod_{i=1}^{n}\left(N-1+(r+1) k_{i}\right) !}} \\
& =\sqrt{\frac{\prod_{j=1}^{s}(N-1+r s+j)}{\prod_{i=1}^{n}\left(\prod_{j=1}^{k_{i}}\left(N-1+r k_{i}+j\right)\right)}} \\
& =\sqrt{\frac{(r s)^{s}+o\left(r^{s}\right)}{\left(\prod_{i=1}^{n}\left(r k_{i}\right)^{k_{i}}\right)+o\left(r^{s}\right)}}=\sqrt{\frac{r^{s} s^{s}+o\left(r^{s}\right)}{r^{s}\left(\prod_{i=1}^{n} k_{i}^{k_{i}}\right)+o\left(r^{s}\right)}} .
\end{aligned}
$$


From this we deduce that

$$
\lim _{r \rightarrow \infty} \frac{a_{r+1}}{a_{r}}=\sqrt{\frac{\left(k_{1}+\cdots+k_{n}\right)^{\left(k_{1}+\cdots+k_{n}\right)}}{k_{1}^{k_{1}} \cdots k_{n}^{k_{n}}}} .
$$

Hence

$$
\begin{aligned}
\lim _{r \rightarrow \infty} \sqrt[r]{a_{r}} & =\lim _{r \rightarrow \infty} \sqrt[2 r]{\frac{((N-1) !)^{n-1}\left(N-1+r \sum_{i=1}^{n} k_{i}\right) !}{\prod_{i=1}^{n}\left(N-1+r k_{i}\right) !}} \\
& =\sqrt{\frac{\left(k_{1}+\cdots+k_{n}\right)^{\left(k_{1}+\cdots+k_{n}\right)}}{k_{1}^{k_{1}} \cdots k_{n}^{k_{n}}}} .
\end{aligned}
$$

Because of Remark 3.1, letting $r \rightarrow \infty$ in inequality (4.1) we can assert that

$$
\left\|P_{1}\right\|_{\mathcal{P}} \cdots\left\|P_{n}\right\|_{\mathcal{P}} \leq \sqrt{\frac{\left(k_{1}+\cdots+k_{n}\right)^{\left(k_{1}+\cdots+k_{n}\right)}}{k_{1}^{k_{1}} \cdots k_{n}^{k_{n}}}}\left\|P_{1} \cdots P_{n}\right\|_{\mathcal{P}} .
$$

Remark 4.2. The bound proved above is independent of the number of variables. If $n \leq N$, then the inequality is sharp. Given any set of natural numbers $\left\{k_{1}, \ldots, k_{n}\right\}$, if we define the polynomials $P_{i}(z)=z_{i}^{k_{i}}$ for $1 \leq i \leq n$, then

$$
\left\|P_{1}\right\|_{\mathcal{P}} \cdots\left\|P_{n}\right\|_{\mathcal{P}}=\sqrt{\frac{\left(k_{1}+\cdots+k_{n}\right)^{\left(k_{1}+\cdots+k_{n}\right)}}{k_{1}^{k_{1}} \cdots k_{n}^{k_{n}}}}\left\|P_{1} \cdots P_{n}\right\|_{\mathcal{P}} .
$$

Clearly, $\left\|P_{i}\right\|_{\mathcal{P}}=1$ for all $1 \leq i \leq n$. On the other hand,

$$
\sup _{\|z\|=1}\left|z_{1}^{k_{1}} \cdots z_{n}^{k_{n}}\right|=\max \left\{x_{1}^{k_{1}} \cdots x_{n}^{k_{n}}:\left\{x_{i}\right\}_{i=1}^{n} \subset \mathbb{R}_{\geq 0} \wedge \sum_{i=1}^{n} x_{i}^{2}=1\right\} .
$$

Applying Lagrange multipliers, the maximum of this function is attained at a point $x$ whose coordinates are $x_{i}=\sqrt{k_{i} /\left(k_{1}+\cdots+k_{n}\right)}$ for $1 \leq i \leq n$ and $x_{i}=0$ for $n<i \leq N$. Therefore

$$
\left\|P_{1} \cdots P_{n}\right\|_{\mathcal{P}}=\sqrt{\frac{k_{1}^{k_{1}} \cdots k_{n}^{k_{n}}}{\left(k_{1}+\cdots+k_{n}\right)^{\left(k_{1}+\cdots+k_{n}\right)}}} .
$$

In order to extend this result to the infinite dimensional setting we need the following lemma.

Lemma 4.3. Let $Q \in \mathcal{P}\left({ }^{m} H\right)$ and let $\left\{e_{i}\right\}_{i=1}^{\infty}$ be an orthonormal basis for $H$. If we define the sequence of polynomials

$$
\begin{gathered}
\widetilde{Q_{l}}: \mathbb{C}^{l} \rightarrow \mathbb{C} \\
\widetilde{Q_{l}}\left(z_{1}, \ldots, z_{l}\right)=Q\left(\sum_{i=1}^{l} z_{i} e_{i}\right),
\end{gathered}
$$

then $\left\|\widetilde{Q_{l}}\right\|_{\mathcal{P}} \underset{l \rightarrow \infty}{\longrightarrow}\|Q\|_{\mathcal{P}}$. 
Proof. It is clear that $\left\|\widetilde{Q_{l}}\right\|_{\mathcal{P}} \leq\|Q\|_{\mathcal{P}}$ for all $l \in \mathbb{N}$. Let us prove the converse inequality. Given any $\varepsilon>0$, choose a unit vector $z \in H$ such that $|Q(z)|>\|Q\|_{\mathcal{P}}-\varepsilon$ and let $z_{i}=\left\langle z, e_{i}\right\rangle$ for $i \geq 1$. Since $\sum_{i=1}^{\infty}\left|z_{i}\right|^{2}=\|z\|^{2}=1$, we have

$$
\left\|\widetilde{Q_{l}}\right\|_{\mathcal{P}} \geq\left|\widetilde{Q_{l}}\left(z_{1}, \ldots, z_{l}\right)\right|=\left|Q\left(\sum_{i=1}^{l}\left\langle z, e_{i}\right\rangle e_{i}\right)\right| .
$$

By continuity, since $\lim _{l \rightarrow \infty} \sum_{i=1}^{l}\left\langle z, e_{i}\right\rangle e_{i}=z$,

$$
\lim _{l \rightarrow \infty}\left|Q\left(\sum_{i=1}^{l}\left\langle z, e_{i}\right\rangle e_{i}\right)\right|=|Q(z)|>\|Q\|_{\mathcal{P}}-\varepsilon .
$$

Hence, there exists $l_{0} \in \mathbb{N}$ such that

$$
\|Q\|_{\mathcal{P}}-\frac{\varepsilon}{2}<\left\|\widetilde{Q_{l}}\right\|_{\mathcal{P}} \leq\|Q\|_{\mathcal{P}}
$$

for all $l \geq l_{0}$.

We are now ready to prove a sharp lower bound for the norm of the product of continuous homogeneous polynomials defined on a complex Hilbert space $H$.

Theorem 4.4. Let $P_{i} \in \mathcal{P}\left({ }^{k_{i}} H\right)$ for $1 \leq i \leq n$. Then

$$
\left\|P_{1}\right\|_{\mathcal{P}} \cdots\left\|P_{n}\right\|_{\mathcal{P}} \leq \sqrt{\frac{\left(k_{1}+\cdots+k_{n}\right)^{\left(k_{1}+\cdots+k_{n}\right)}}{k_{1}^{k_{1}} \cdots k_{n}^{k_{n}}}}\left\|P_{1} \cdots P_{n}\right\|_{\mathcal{P}}
$$

and this inequality is sharp.

Proof. As we did in Lemma 4.3, for $l \in \mathbb{N}$ and $1 \leq i \leq n$, let us define

$$
\begin{gathered}
\widetilde{P_{l, i}}: \mathbb{C}^{l} \rightarrow \mathbb{C} \\
\widetilde{P_{l, i}}\left(z_{1}, \ldots, z_{l}\right)=P_{i}\left(\sum_{j=1}^{l} z_{j} e_{j}\right) .
\end{gathered}
$$

From Theorem 4.1 we know that

$$
\left\|\widetilde{P_{l, 1}}\right\|_{\mathcal{P}} \cdots\left\|\widetilde{P_{l, n}}\right\|_{\mathcal{P}} \leq \sqrt{\frac{\left(k_{1}+\cdots+k_{n}\right)^{\left(k_{1}+\cdots+k_{n}\right)}}{k_{1}^{k_{1}} \cdots k_{n}^{k_{n}}}}\left\|\widetilde{P_{l, 1}} \cdots \widetilde{P_{l, n}}\right\|_{\mathcal{P}}
$$

We may now let $l \rightarrow \infty$, and the result follows by Lemma 4.3 .

The ideas used in Remark 4.2 allow us to see that given $\left\{k_{i}\right\}_{i=1}^{n} \subset \mathbb{N}$, if we define the polynomials $P_{i}(z)=\left\langle z, e_{i}\right\rangle^{k_{i}}$ for $1 \leq i \leq n$, then

$$
\left\|P_{1}\right\|_{\mathcal{P}} \cdots\left\|P_{n}\right\|_{\mathcal{P}}=\sqrt{\frac{\left(k_{1}+\cdots+k_{n}\right)^{\left(k_{1}+\cdots+k_{n}\right)}}{k_{1}^{k_{1}} \cdots k_{n}^{k_{n}}}}\left\|P_{1} \cdots P_{n}\right\|_{\mathcal{P}} .
$$

Thus this bound is sharp.

It is worth pointing out that working on Hilbert spaces the isomorphism $P \mapsto \check{P}$ is an isometry between $\mathcal{P}\left({ }^{m} H\right)$ and $\mathcal{L}^{s}\left({ }^{m} H\right)$ (see for instance [5] and [20]). We may use this fact to derive an inequality for continuous symmetric $m$-linear mappings from $H^{m}$ to $\mathbb{C}$. 
Given $\Phi_{j} \in \mathcal{L}^{s}\left({ }^{k_{j}} H\right)$ for $1 \leq j \leq n$, set $k=\sum_{j=1}^{n} k_{j}$. Let us define the symmetrized product $\left(\Phi_{1} \cdots \Phi_{n}\right)_{s}\left(z_{1}, \ldots, z_{k}\right)$ of $\left\{\Phi_{j}\right\}_{j=1}^{n}$ by

$\frac{1}{k !} \sum_{\tau \in \mathcal{G}_{k}} \Phi_{1}\left(z_{\tau(1)}, \ldots, z_{\tau\left(k_{1}\right)}\right) \Phi_{2}\left(z_{\tau\left(k_{1}+1\right)}, \ldots, z_{\tau\left(k_{1}+k_{2}\right)}\right) \cdots \Phi_{n}\left(z_{\tau\left(k-k_{n}+1\right)}, \ldots, z_{\tau(k)}\right)$, where $\mathcal{G}_{k}$ denotes the permutation group of $k$ elements.

Corollary 4.5. Let $\Phi_{j} \in \mathcal{L}^{s}\left({ }^{k_{j}} H\right)$ for $1 \leq j \leq n$. Then

$$
\left\|\Phi_{1}\right\|_{\mathcal{L}^{s}} \cdots\left\|\Phi_{n}\right\|_{\mathcal{L}^{s}} \leq \sqrt{\frac{\left(k_{1}+\cdots+k_{n}\right)^{\left(k_{1}+\cdots+k_{n}\right)}}{k_{1}^{k_{1}} \cdots k_{n}^{k_{n}}}}\left\|\left(\Phi_{1} \cdots \Phi_{n}\right)_{s}\right\|_{\mathcal{L}^{s}}
$$

and this inequality is sharp.

The first step in obtaining these sharp inequalities on complex Hilbert spaces was taken in Proposition 2.4. We do not have a similar argument for the real case. However, if $K$ is a real Hilbert space, we can give estimates for the norm of products of polynomials or symmetric multilinear mappings using the natural complexification of $K$ (see for instance [40, pp. 313-314] and [32] for more details).

Corollary 4.6. Let $P_{j} \in \mathcal{P}\left({ }^{k_{j}} K\right)$ for $1 \leq j \leq n$. Then

$$
\left\|P_{1}\right\|_{\mathcal{P}} \cdots\left\|P_{n}\right\|_{\mathcal{P}} \leq 2^{\left[\left(k_{1}+\cdots+k_{n}\right)-2\right] / 2} \sqrt{\frac{\left(k_{1}+\cdots+k_{n}\right)^{\left(k_{1}+\cdots+k_{n}\right)}}{k_{1}^{k_{1}} \cdots k_{n}^{k_{n}}}}\left\|P_{1} \cdots P_{n}\right\|_{\mathcal{P}} .
$$

Proof. Let $H=K \oplus i K$ be the natural complexification of $K$. Given $P_{j} \in \mathcal{P}\left({ }^{k_{j}} K\right)$ for $1 \leq j \leq n$, let $Q_{j} \in \mathcal{P}\left({ }^{k_{j}} H\right)$ be the unique complex extension of $P_{j}$. It is known that (see [32, Prop. 19])

$$
\left\|P_{j}\right\|_{\mathcal{P}} \leq\left\|Q_{j}\right\|_{\mathcal{P}}=\sup _{\|x+i y\|_{H}=1}\left|Q_{j}(x+i y)\right| \leq 2^{\left(k_{j}-2\right) / 2}\left\|P_{j}\right\|_{\mathcal{P}} .
$$

Therefore

$$
\begin{aligned}
\left\|P_{1}\right\|_{\mathcal{P}} \cdots\left\|P_{n}\right\|_{\mathcal{P}} & \leq\left\|Q_{1}\right\|_{\mathcal{P}} \cdots\left\|Q_{n}\right\|_{\mathcal{P}} \leq \sqrt{\frac{\left(k_{1}+\cdots+k_{n}\right)^{\left(k_{1}+\cdots+k_{n}\right)}}{k_{1}^{k_{1}} \cdots k_{n}^{k_{n}}}}\left\|Q_{1} \cdots Q_{n}\right\|_{\mathcal{P}} \\
& \leq 2^{\left[\left(k_{1}+\cdots+k_{n}\right)-2\right] / 2} \sqrt{\frac{\left(k_{1}+\cdots+k_{n}\right)^{\left(k_{1}+\cdots+k_{n}\right)}}{k_{1}^{k_{1}} \cdots k_{n}^{k_{n}}}}\left\|P_{1} \cdots P_{n}\right\|_{\mathcal{P}} .
\end{aligned}
$$

Remark 4.7. Let $\phi_{j}: \mathbb{R}^{n} \rightarrow \mathbb{R}$ be linear functionals for $1 \leq j \leq n \leq 5$. In [33, A. Pappas and S. G. Révész proved that

$$
\left\|\phi_{1}\right\|_{\mathcal{P}} \cdots\left\|\phi_{n}\right\|_{\mathcal{P}} \leq n^{n / 2}\left\|\phi_{1} \cdots \phi_{n}\right\|_{\mathcal{P}} .
$$

Consequently, it is clear that we cannot expect a sharp inequality in Corollary 4.6.

Finally, we give a real version of Corollary 4.5 .

Corollary 4.8. Let $\Phi_{j} \in \mathcal{L}^{s}\left({ }^{k_{j}} K\right)$ for $1 \leq j \leq n$. Then

$$
\left\|\Phi_{1}\right\|_{\mathcal{L}^{s}} \cdots\left\|\Phi_{n}\right\|_{\mathcal{L}^{s}} \leq 2^{\left[\left(k_{1}+\cdots+k_{n}\right)-2\right] / 2} \sqrt{\frac{\left(k_{1}+\cdots+k_{n}\right)^{\left(k_{1}+\cdots+k_{n}\right)}}{k_{1}^{k_{1}} \cdots k_{n}^{k_{n}}}}\left\|\left(\Phi_{1} \cdots \Phi_{n}\right)_{s}\right\|_{\mathcal{L}^{s}} .
$$




\section{Products of Bounded linear forms on COMPlex Hilbert spaces}

Given a Banach space $E$, let $E^{\prime}$ denote its dual space. In [13], the authors defined the $n$th linear polarization constant of $E$ by

$c_{n}(E)=\inf \left\{M>0:\left\|\phi_{1}\right\|_{E^{\prime}} \cdots\left\|\phi_{n}\right\|_{E^{\prime}} \leq M\left\|\phi_{1} \cdots \phi_{n}\right\|_{\mathcal{P}}\right.$, for all $\left.\phi_{1}, \ldots, \phi_{n} \in E^{\prime}\right\}$

and the linear polarization constant of $E$ by $c(E)=\lim \sup c_{n}^{1 / n}(E)$. Also, in [37], it was proved that given $n \in \mathbb{N}$, then infinite dimensional Hilbert spaces have the smallest $n$th polarization constant.

If $K$ is a real Hilbert space it was conjectured in [13] that $c_{n}(K)=n^{n / 2}$. As we mentioned earlier, a positive answer was given in 33 . for $n \leq 5$, but the remaining cases are still open problems. For complex Hilbert spaces this conjecture is true. The following theorem is due to J. Arias-de-Reyna.

Theorem 5.1 ([2, Theorem 4]). Let $\left\{z_{k}\right\}_{k=1}^{n}$ be unit vectors in a complex Hilbert space $H$. There is a unit vector $z \in H$ such that $\left|\left\langle z, z_{1}\right\rangle \cdots\left\langle z, z_{n}\right\rangle\right| \geq n^{-n / 2}$.

In the proof, the author showed that $n^{-n / 2}$ is the best possible constant because for any orthonormal system $\left\{z_{k}\right\}_{k=1}^{n}$,

$$
\sup _{\|z\|=1}\left|\left\langle z, z_{1}\right\rangle \cdots\left\langle z, z_{n}\right\rangle\right|=n^{-n / 2}
$$

However, he did not prove that the equality holds only for orthonormal systems.

Note that for linear functionals on a complex Hilbert space the inequality obtained in Theorem 4.4 is the same one that was proved by J. Arias-de-Reyna. Since our inequality allows us to handle nonlinear factors, we can use it to prove that the orthonormality of the set $\left\{z_{k}\right\}_{k=1}^{n}$ is a necessary condition to achieve this minimum value. In the following lemma we will prove this assertion for the special case when $n=2$. This particular result will be needed for the general case.

Lemma 5.2. Let $z_{1}, z_{2}$ be unit vectors in a complex Hilbert space. If

$$
\sup _{\|z\|=1}\left|\left\langle z, z_{1}\right\rangle\left\langle z, z_{2}\right\rangle\right|=\frac{1}{2}
$$

then $\left\langle z_{1}, z_{2}\right\rangle=0$.

Proof. First, note that $\left|\left\langle z, z_{1}\right\rangle\left\langle z, z_{2}\right\rangle\right|=\left|\left\langle z, z_{1}\right\rangle\left\langle z,-z_{2}\right\rangle\right|$. Hence we can assume, without loss of generality, that $\Re\left\langle z_{1}, z_{2}\right\rangle \geq 0$.

In order to have a lower estimate for the supremum we want to compute

$$
\begin{aligned}
\left|\left\langle\frac{z_{1}+z_{2}}{\left\|z_{1}+z_{2}\right\|}, z_{1}\right\rangle\left\langle\frac{z_{1}+z_{2}}{\left\|z_{1}+z_{2}\right\|}, z_{2}\right\rangle\right| & =\frac{\left|\left(1+\left\langle z_{2}, z_{1}\right\rangle\right)\left(1+\left\langle z_{1}, z_{2}\right\rangle\right)\right|}{\left\langle z_{1}+z_{2}, z_{1}+z_{2}\right\rangle} \\
& =\frac{\left|1+\left\langle z_{1}, z_{2}\right\rangle\right|^{2}}{2+2 \Re\left\langle z_{1}, z_{2}\right\rangle} .
\end{aligned}
$$


Thus we obtain

$$
\begin{aligned}
\sup _{\|z\|=1}\left|\left\langle z, z_{1}\right\rangle\left\langle z, z_{2}\right\rangle\right| & \geq\left|\left\langle\frac{z_{1}+z_{2}}{\left\|z_{1}+z_{2}\right\|}, z_{1}\right\rangle\left\langle\frac{z_{1}+z_{2}}{\left\|z_{1}+z_{2}\right\|}, z_{2}\right\rangle\right| \\
& =\frac{\left[1+\Re\left\langle z_{1}, z_{2}\right\rangle\right]^{2}+\left[\Im\left\langle z_{1}, z_{2}\right\rangle\right]^{2}}{2+2 \Re\left\langle z_{1}, z_{2}\right\rangle} \\
& =\frac{1}{2}\left(1+\Re\left\langle z_{1}, z_{2}\right\rangle+\frac{\left[\Im\left\langle z_{1}, z_{2}\right\rangle\right]^{2}}{1+\Re\left\langle z_{1}, z_{2}\right\rangle}\right),
\end{aligned}
$$

which is strictly greater than $1 / 2$ unless $\left\langle z_{1}, z_{2}\right\rangle=0$.

Finally, we can prove the general case.

Theorem 5.3. Let $\left\{z_{k}\right\}_{k=1}^{n}$ be unit vectors in a complex Hilbert space $H$. If

$$
\sup _{\|z\|=1}\left|\left\langle z, z_{1}\right\rangle \cdots\left\langle z, z_{n}\right\rangle\right|=n^{-n / 2},
$$

then $\left\{z_{k}\right\}_{k=1}^{n}$ is an orthonormal system.

Proof. Let $P_{k}(z)=\left\langle z, z_{k}\right\rangle$ for $1 \leq k \leq n$, and $P=P_{1} \cdots P_{n}$. We will show that $\left\langle z_{n-1}, z_{n}\right\rangle=0$, but the same idea will give us $\left\langle z_{i}, z_{j}\right\rangle=0$ for any $1 \leq i<j \leq n$. We have

$$
\begin{aligned}
1 & =\left\|P_{1}\right\|_{\mathcal{P}} \cdots\left\|P_{n}\right\|_{\mathcal{P}}=\sqrt{n^{n}}\|P\|_{\mathcal{P}} \\
& \geq \sqrt{n^{n}} \sqrt{\frac{2^{2}}{n^{n}}}\left\|P_{1}\right\|_{\mathcal{P}} \cdots\left\|P_{n-2}\right\|_{\mathcal{P}}\left\|P_{n-1} P_{n}\right\|_{\mathcal{P}} \\
& =2\left\|P_{n-1} P_{n}\right\|_{\mathcal{P}} \geq 2 \sqrt{\frac{1}{2^{2}}}\left\|P_{n-1}\right\|_{\mathcal{P}}\left\|P_{n}\right\|_{\mathcal{P}}=1 .
\end{aligned}
$$

In particular, $\left\|P_{n-1} P_{n}\right\|_{\mathcal{P}}=1 / 2$ and so, from Lemma 5.2, we conclude that $\left\langle z_{n-1}, z_{n}\right\rangle=0$.

\section{ACKNOWLEDGEMENTS}

The author wishes to express his gratitude to D. Carando, V. Dimant, D. Galicer, S. Lassalle, S. Muro and I. Zalduendo for several helpful comments during the preparation of this paper.

\section{REFERENCES}

1. V. A. Anagnostopoulos and S. G. Révész, Polarization constants for products of linear functionals over $\mathbb{R}^{2}$ and $\mathbb{C}^{2}$ and Chebyshev constants of the unit sphere. Publ. Math. Debrecen 68 (1-2) (2006), pp. 63-75. MR.2213542 (2006m:46054)

2. J. Arias-de-Reyna, Gaussian variables, polynomials and permanents. Linear Algebra Appl. 285 (1998), pp. 107-114. MR1653495(2000a:15008)

3. G. Aumann, Satz über das Verhalten von Polynomen auf Kontinuen. Sitz. Preuss. Akad. Wiss. Phys.-Math. Kl. (1933), 926-931.

4. K. M. Ball, The complex plank problem. Bull. London Math. Soc. 33 (4) (2001), pp. 433-442. MR:1832555 (2002b:46023)

5. S. Banach, Über Homogene Polynome in $L^{2}$. Studia Math. 7 (1938), pp. 36-44.

6. B. Beauzamy, Extremal products in Bombieri's norm. Rend. Istit. Mat. Univ. Trieste, Suppl. Vol. 28 (1997), pp. 73-89. MR.1602318 (99a:11028) 
7. B. Beauzamy, Products of many-variable polynomials: pairs that are maximal in Bombieri's norm. J. Number Theory 55 (1) (1995), pp. 129-143. MR.1361564 (97a:11038)

8. B. Beauzamy, Products of polynomials and a priori estimates for coefficients in polynomial decompositions: a sharp result. J. Symbolic Comput. 13 (5) (1992), pp. 463-472. MR1170091 (93h:11140)

9. B. Beauzamy, E. Bombieri, P. Enflo and H. L. Montgomery, Products of polynomials in many variables. J. Number Theory 36 (2) (1990), pp. 219-245. MR.1072467 (91m:11015)

10. B. Beauzamy and J. Dégot, Differential identities. Trans. Amer. Math. Soc. 347 (7) (1995), pp. 2607-2619. MR1277095 (96c:05009)

11. B. Beauzamy and P. Enflo, Estimations de produits de polynômes. J. Number Theory 21 (3) (1985), pp. 390-412. MR814012 (87b:42001)

12. B. Beauzamy, J.-L. Frot and C. Millour, Massively parallel computations on many-variable polynomials. Ann. Math. Artificial Intelligence 16 (1) (1996), pp. 251-283. MR1389850 (98c:68078)

13. C. Benítez, Y. Sarantopoulos and A. Tonge, Lower bounds for norms of products of polynomials. Math. Proc. Cambridge Philos. Soc. 124 (3) (1998), pp. 395-408. MR.1636556|(99h:46077)

14. E. Bombieri and J. Vaaler, On Siegel's lemma. Invent. Math. 73 (1) (1983), pp. 11-32. MR.707346 (85g:11049a)

15. P. B. Borwein, Exact inequalities for the norms of factors of polynomials. Canad. J. Math. 46 (4) (1994), pp. 687-698. MR.1289054(95k:26015)

16. C. Boyd and R. Ryan, The norm of the product of polynomials in infinite dimensions. Proc. Edinb. Math. Soc. (2) 49 (1) (2006), pp. 17-28. MR.2202139 (2006i:46064)

17. D. W. Boyd, Bounds for the height of a factor of a polynomial in terms of Bombieri's norms I. The largest factor. J. Symbolic Comput. 16 (2) (1993), pp. 115-130. MR.1253908(94m:11032a)

18. D. W. Boyd, Bounds for the height of a factor of a polynomial in terms of Bombieri's norms II. The smallest factor. J. Symbolic Comput. 16 (2) (1993), pp. 131-145. MR1253909 (94m:11032b)

19. D. W. Boyd, Sharp inequalities for the product of polynomials. Bull. London Math. Soc. 26 (5) (1994), pp. 449-454. MR.1308361 (95m:30008)

20. S. Dineen, Complex analysis on infinite dimensional spaces. Springer-Verlag, London, 1999. MR.1705327 (2001a:46043)

21. J. D. Donaldson and Q. I. Rahman, Inequalities for polynomials with a prescribed zero. Pacific J. Math. 41 (1972), pp. 375-378. MR0308371(46:7485)

22. P. E. Frenkel, Pfaffians, Hafnians and products of real linear functionals. Math. Res. Lett. 15 (2) (2008), pp. 351-358. MR2385646 (2008m:15016)

23. J. C. García-Vázquez and R. Villa, Lower bounds for multilinear forms defined on Hilbert spaces. Mathematika 46 (2) (1999), pp. 315-322. MR1832622 (2002c:46045)

24. A. O. Gel'fond, Transcendental and algebraic numbers. (Translation by L. F. Boron of the 1952 Russian edition), Dover, New York, 1960. MR0111736 (22:2598)

25. J.-P. Kahane, Séries de Fourier absolument convergentes. Springer-Verlag, New York, 1970. MR0275043 (43:801)

26. H. Kneser, Das Maximum des Produkts zweier Polynome. Sitz. Preuss. Akad. Wiss. Phys.Math. Kl. (1934), pp. 429-431.

27. A. E. Litvak, V. D. Milman and G. Schechtman, Averages of norms and quasi-norms. Math. Ann. 312 (1) (1998), pp. 95-124. MR.1645952(2000c:46013)

28. K. Mahler, An application of Jensen's formula to polynomials. Mathematika 7 (1960), pp. 98-100. MR0124467 (23:A1779)

29. K. Mahler, On some inequalities for polynomials in several variables. J. London Math. Soc. 37 (1962), pp. 341-344. MR0138593 (25:2036)

30. M. Matolcsi, A geometric estimate on the norm of product of functionals. Linear Algebra Appl. 405 (2005), pp. 304-310. MR2148177 (2006d:46005b)

31. M. Matolcsi, The linear polarization constant of $\mathbb{R}^{n}$. Acta Math. Hungar. 108 (1-2) (2005), pp. 129-136. MR2155246 (2006d:46005a)

32. G. A. Muñoz, Y. Sarantopoulos and A. Tonge, Complexifications of real Banach spaces, polynomials and multilinear maps. Stud. Math. 134 (1) (1999), pp. 1-33. MR 1688213 (2000g:46009)

33. A. Pappas and S. G. Révész, Linear polarization constants of Hilbert spaces. J. Math. Anal. Appl. 300 (1) (2004), pp. 129-146. MR2100242(2005h:46072) 
34. D. Pinasco and I. Zalduendo, Integral representation of holomorphic functions on Banach spaces. J. Math. Anal. Appl. 308 (1) (2005), pp. 159-174. MR2142411 (2006d:46053)

35. I. E. Pritsker and S. Ruscheweyh, Inequalities for products of polynomials I. Math. Scand. 104 (1) (2009), pp. 147-160. MR2498378 (2010f:30006)

36. I. E. Pritsker and S. Ruscheweyh, Inequalities for products of polynomials II. Aequationes Math. 77 (1-2) (2009), pp. 119-132. MR2495722 (2010c:30009)

37. S. G. Révész and Y. Sarantopoulos, Plank problems, polarization and Chebyshev constants. Satellite Conference on Infinite Dimensional Function Theory. J. Korean Math. Soc. 41 (1) (2004), pp. 157-174. MR2048707 (2004m:46033)

38. R. Ryan and B. Turett, Geometry of spaces of polynomials. J. Math. Anal. Appl. 221 (2) (1998), pp. 698-711. MR1621703 (99g:46015)

39. B. Reznick, An inequality for products of polynomials, Proc. Amer. Math. Soc. 117 (4) (1993), pp. 1063-1073. MR1119265 (93e:11058)

40. A. E. Taylor, Additions to the theory of polynomials in normed linear spaces. Tohoku Math. J. 44 (1938), pp. 302-318.

Departamento de Matemáticas y Estadística, Universidad Torcuato di Tella, Miñones 2177 (C1428ATG), Ciudad Autónoma de Buenos Aires, Argentina - and - CONICET

E-mail address: dpinasco@utdt.edu 